\title{
Power Management in Multiuser Adaptive Modulation Transmission under QoS Requirements
}

\author{
Saud Althunibat, ${ }^{1}$ Nizar Zorba, ${ }^{2}$ Charalabos Skianis, ${ }^{3}$ and Christos Verikoukis ${ }^{4}$ \\ ${ }^{1}$ University of Jordan, Amman 11942, Jordan \\ ${ }^{2}$ Qatar Mobility Innovations Center (QMIC), Doha 210531, Qatar \\ ${ }^{3}$ University of the Aegean, 83200 Samos, Greece \\ ${ }^{4}$ CTTC, 08860 Barcelona, Spain
}

Correspondence should be addressed to Nizar Zorba; nizarz@qmic.com

Received 25 April 2013; Accepted 25 July 2013

Academic Editor: Athanasios Kanatas

Copyright (C) 2013 Saud Althunibat et al. This is an open access article distributed under the Creative Commons Attribution License, which permits unrestricted use, distribution, and reproduction in any medium, provided the original work is properly cited.

A transmitter power management mechanism is presented in this paper where Multiple-Input Multiple-Output (MIMO) Multiuser Random Beamforming with Adaptive Modulation strategy is performed by the system. The objective of the proposed mechanism is quality of service (QoS) satisfaction for the scheduled user. The QoS is represented by the application demanded data rate and symbol error rate. The power outage problem is considered and a practical method to minimize the outage probability is proposed. The obtained results are encouraging as they show a great decrease in the system power budget. The multiuser system capability is also exploited to achieve larger power saving values and smaller probability of power outage by scheduling the user with the best channel characteristics at each time instant. The amount of saved power and the power outage probability are both presented through closed-form expressions. These theoretical results are compared to computer simulations, which show very good agreement in performance.

\section{Introduction}

Power efficiency in wireless systems is a very interesting and timely topic in the research arena. The wireless network interface consumes a significant amount of power that is continuously increasing mainly due to the transmitter operation at the base station (BS). Wireless operators consume a huge amount of power where the electric bill constitutes a large portion of the network running costs [1]. Therefore, if we decrease the consumed power in BSs, we will reduce the communication costs, and we will help in the environmental care by reducing the $\mathrm{CO}_{2}$ emissions.

The main characteristic of the wireless channel is its variability over time, where several approaches have been implemented to tackle such channel variations. A strategy that has been already implemented in realistic systems is Adaptive Modulation (AM). By employing AM, the transmitter is continuously changing the employed modulation to match with the instantaneous channel conditions. AM is shown as a way to increase the system performance [2].

Such employment of the AM is mainly devoted to increase the average data rate in the system, but other system indicators, rather than the data rate, are also interesting to the system operator. One of such objectives relates to the requested quality of service (QoS) of the customers within the network. A potential measure of the QoS is through the minimum guaranteed data rate with the maximum allowed symbol error rate (SER) per user. Each served user is guaranteed a minimum signal-to-noise ratio (SNR), which provides its demanded rate and allows the user to properly decode its intended data with the predefined SER [3]. Regarding the minimum requirement per user, previous studies have shown that the user satisfaction is insignificantly increased by a performance higher than its demands, while on the other hand, if the provided resources fail to guarantee its requirements, the satisfaction drastically decreases [4]. 
Thus, an attractive transmission scheme is accomplished by meeting the minimum requirements for each scheduled user while minimizing the total transmitted power.

The availability of multiple antennas at the BS allows the implementation of multiple input multiple-output (MIMO) beamforming schemes at the transmitter side. To extract all the benefit from MIMO, awareness of the channel state information (CSI) is required at the transmitter side before the transmission starts. However, the required overhead to enable CSI is huge and impractical, especially in multiuser scenarios [3]. Partial CSI is the appropriate mechanism to enable beamforming with a feasible overhead on the system resources [5]. Multiuser random beamforming is an interesting beamforming strategy that only needs partial CSI and it provides an outstanding performance [5] by selecting, at each scheduling time, the user showing the best performance with respect to a randomly generated beam at the transmitter side. It remains to tackle its performance under QoS restrictions [3] in order to benefit from the scenario characteristics in the QoS achievement.

A cross-layer (XL) strategy will be proposed to employ information from the channel characteristics in the QoS management. Moreover, an XL power saving philosophy is regarded; as for a given QoS per user the system will be allowed to decrease the transmitted power by a continuous monitoring of the channel conditions. Therefore, it will be aware of the exact required power to meet the QoSrequirement of each user, with the consequent decrease in the overall transmitted power.

The minimization of the amount of transmitted power, while the QoS is achieved, is a challenge to system designers because of the nature of the wireless channel and the limitations on the power and spectrum resources. Moreover, the diverse requirements of QoS complicate the task, as several applications can coexist in the system at the same time. Several solutions have been presented in the literature to separately solve each research issue; $[6,7]$ propose adaptive subcarrier-bit allocation based on the QoS requirements while $[8,9]$ consider the QoS problem through admission control and scheduling algorithms. A power allocation over transmitting beams in MIMO system is presented in [10], where the required rate for each scheduled user is guaranteed. The rate maximization and QoS requirements are combined in [11] as the design objectives; and on the other hand, a proportional fair scheduler to tackle with different QoS requirement is designed in [12].

In the current paper, a power management mechanism based on the AM technique is presented for the MIMO Multiuser Random Beamforming scheme, where the user with the best channel characteristics is scheduled for transmission, and then the modulation is employed on the basis of its QoSrequirements represented by the demanded data rate and SER values. The transmitted power is allocated to the minimum value that satisfies the required QoS, obtaining a cross-layer framework to achieve both rate and SER demands. The required transmit power is presented in a closed-form expression, together with its statistical distributions. An important metric to identify the system performance in practical systems is the power outage [13] represented by the probability of exceeding the maximum allowed transmitted power. This metric is also obtained in a closed-form expression, where no previous contribution in the literature has obtained all these quantities through mathematical formulations. A practical solution of the power outage problem is presented, through a modification of the opportunistic beamforming decision to select another user that can be served without power outage probability. Computer simulations will validate the mathematically obtained results.

The remainder of this paper is organized as follows. Section 2 presents the system model, the MIMO opportunistic beamforming at the transmitter side, and a review of the Adaptive Modulation procedure. Section 3 discusses the proposed power management technique and its performance through a closed-form expression, followed by Section 4 with the discussion of the resultant resource power outage through the mathematical expressions. Section 5 proposes a modified opportunistic beamforming to overcome the power outage problem, followed by Section 6 with numerical results and simulations. The paper finally draws the conclusions in Section 7.

\section{System Model}

We focus on the downlink channel where $K$ receivers, each one of them equipped with a single receiving antenna, are being served by a transmitter at the base station (BS) provided with $n_{t}$ transmitting antennas. A channel $\mathbf{h}(t)_{\left[1 \times n_{t}\right]}$ is considered between each user and the BS where a quasi static block fading model is assumed. The channel is kept constant through the coherence time and independently changes between consecutive time intervals with independent and identically distributed (i.i.d.) complex Gaussian entries $\sim \mathrm{CN}(0,1)$. Let $\mathbf{w}_{k}(t)_{\left[n_{t} \times 1\right]}$ denotes the transmitted signal to the $k$ th; then the received signal $y_{k}(t)$ is given by

$$
y_{k}(t)=\mathbf{h}_{k}(t) \mathbf{w}_{k}(t)+z_{k}(t),
$$

where $z_{k}(t)$ is an additive i.i.d. complex noise component with zero mean and $E\left\{\left|z_{k}\right|^{2}\right\}=\sigma^{2}$. A total transmission power of $P_{T}$ is considered. To transmit the signal from the $n_{t}$ antennas, a beamforming vector $\mathbf{g}_{k}(t)_{\left[n_{t} \times 1\right]}$ is required at the transmitter side, making the transmitted signal $\mathbf{w}_{k}(t)=\mathbf{g}_{k}(t) s_{k}(t)$, where $s_{k}(t)$ is the uncorrelated data symbol to the $k$ th user with $E\left\{\left|s_{k}\right|^{2}\right\}=1$. To simplify the notation, time index is dropped whenever possible.

We assume a heterogeneous system, where the $K$ users run different applications. Each application has its own QoS requirements represented by a specified data rate and SER.

2.1. Opportunistic Beamforming. A main beamforming policy in multiuser MIMO scenarios is the random beamforming [5], where the transmitter accomplishes a maximization of the system average data rate. During the acquisition step, an i.i.d. complex Gaussian $\sim \mathrm{CN}(0,1)$ unit-power random beam $\mathbf{g}_{\left[n_{t} \times 1\right]}$ is generated at the transmitter side and a known training sequence is transmitted using the generated beam for all the users in the system, and each user calculates the received SNR and feeds it back to the BS. The BS scheduler 
chooses the user with the highest SNR value to benefit from its current channel situation, leading to improve the global system performance. As the user with the best channel conditions is selected for transmission, this scheme is known as the Opportunistic Scheduler [5]. A modified version of this scheduler has been already implemented in the cellular 3.5G HSDPA-HDR standard.

Besides its low complexity, the opportunistic beamforming only needs partial CSI which motivates its inclusion in commercial systems. The achieved throughput (TH) using opportunistic beamforming is given as follows:

$$
\mathrm{TH}=E\left\{\log _{2}\left(1+\max _{1 \leq k \leq K} \mathrm{SNR}_{k}\right)\right\},
$$

where $E\{\cdot\}$ is the expectation operator to denote the average value. Notice that the value of $\max _{1 \leq k \leq K} \mathrm{SNR}_{k}$ reflects the serving SNR (i.e., the SNR that the user $k$ obtains when it is selected for transmission). The SNR value $\gamma_{k}$ calculated at each user as follows:

$$
\gamma_{k}=\frac{P_{T}\left|\mathbf{h}_{k} \mathbf{g} s_{k}\right|^{2}}{\sigma^{2}}=\frac{P_{T}\left|\mathbf{h}_{k}\right|^{2}}{\sigma^{2}},
$$

where the transmitted power value is usually set to unity, except for power saving mechanisms as we will later see in Section 3.

Based on such selection philosophy to deliver service to the users, the serving SNR distribution can be obtained from the SNR probability distribution function (pdf) $b(\gamma)$ of i.i.d. complex Gaussian channels $[5,14]$, which is stated as

$$
b(\gamma)=\sigma^{2} e^{-\left(\gamma \cdot \sigma^{2}\right)},
$$

and its cumulative distribution function (cdf) $B(\gamma)$ is formulated [14] as

$$
B(\gamma)=1-e^{-\left(\gamma \cdot \sigma^{2}\right)}
$$

and since the serving SNR is the maximum over all the users' SNR values, then the cdf of the serving $\operatorname{SNR} F(\gamma)$ is stated as [15]

$$
F(\gamma)=(B(\gamma))^{K}=\left[1-e^{-\left(\gamma \cdot \sigma^{2}\right)}\right]^{K},
$$

and the pdf $f(\gamma)$ of the serving SNR is therefore obtained as

$$
f(\gamma)=K\left[1-e^{-\left(\gamma \cdot \sigma^{2}\right)}\right]^{K-1}\left[\sigma^{2} e^{-\left(\gamma \cdot \sigma^{2}\right)}\right] .
$$

Considering the cdf of the serving rate, the probability $P_{r}$ for the serving SNR to be above some predefined threshold $\gamma^{*}$ is given as

$$
P_{r}=1-\left[1-e^{-\left(\sigma^{2} \cdot \gamma^{*}\right)}\right]^{K},
$$

where the value of $\gamma^{*}$ can be the lowest acceptable SNR value in each modulation step, as now will be explained.
2.2. Adaptive Modulation (AM). The wireless channel is continuously fluctuating. Adaptation to these changes is required to achieve good performance in the wireless systems. The AM strategy [16] is accomplished by an instantaneous change in the employed modulation level to match the BS transmitter parameters to the channel conditions subject to the required $\mathrm{Q} o \mathrm{~S}$ of the served user.

Notice that the selection of the user with the best SNR value is actually another way to adapt the transmitter processing to the channel properties. In this aspect, it follows the same strategy as AM schemes [16], with both strategies looking towards improving the wireless channel performance. This paper concentrates on the opportunistic scheduler and we consider it together with AM for the transmitter adaptation to the channel characteristics. We will later employ this scenario to present a power management strategy in a closed-form expression.

Consider an AM scheme that offers $M$ available rates $\left\{R_{1}, \ldots, R_{M}\right\}$ in ascending order [2]. Each rate will be used for transmission when the QoS guarantee of the served user is implied using the corresponding modulation type. The threshold for any modulation type represents the lowest SNR value that can satisfy the required SER value by the corresponding modulation.

\section{Power Management Mechanism}

As the considered scenario with the AM transmission and the opportunistic beamforming has been discussed, we now present the power management mechanism along with its closed form mathematical expression. The paper will exploit the characteristics of the AM strategy that are defined in terms of intervals.

Once the user with the highest SNR is scheduled, its required rate and SER are determined and the total SNR range is divided into modulation regions according to the required SER. Each region supports one modulation type. To achieve the required rate and SER, the received SNR must be within the corresponding modulation region. After that, the transmitted power $P_{t}$ must be allocated to adjust the SNR to within the modulation region.

Due to the division into regions, in each region the same modulation is employed with the same required SER satisfaction, a matter that we will exploit to achieve power saving. For example, suppose that the QoS of the scheduled user is satisfied by SER $=10^{-3}$ and QPSK modulation; then, according to the SER, the total range is divided into regions. Table 1 presents the modulation thresholds for (SER $=10^{-3}$ ) calculated upon the SER equations in [17]. Based on Table 1, and as the QoS is guaranteed by QPSK modulation, the required data rate is achieved by any SNR value between 10.34 and 14.20. In the case of SNR $=10.34$, however, the allocated power is less than that of the power for $\mathrm{SNR}=14.20$. Hence, for the purpose of power saving, the allocated power must be decreased to make the SNR expression (3) to match the exact SNR threshold (10.34 in our example) of the required modulation. 
TABLE 1: SNR thresholds for SER $=10^{-3}$.

\begin{tabular}{lc}
\hline Modulation type & SNR threshold $\gamma(\mathrm{dB})$ \\
\hline BPSK & 6.78 \\
QPSK & 10.34 \\
8QAM & 14.21 \\
16QAM & 17.62 \\
32QAM & 20.84 \\
64QAM & 23.96 \\
\hline
\end{tabular}

Notice that each application requires one of the $N$ SER values and one of $M$ rates (i.e., modulation types), so the QoS for any application is given by two values $\left(R_{m}, \operatorname{SER}_{n}\right)$, where $(n=1,2, \ldots, N)$ and $(m=1,2, \ldots, M)$. Following the proposed philosophy, the resultant required transmit power, denoted by $P_{x}$, considers the ratio between the measured SNR $(\gamma)$ and the SNR threshold, making it to be formulated as

$$
P_{x}=P_{T} \frac{\gamma_{m, n}}{\gamma}
$$

where $\gamma_{m, n}$ is the threshold of the required modulation type $m$ obtained based on the required $\mathrm{SER}_{n}$. For the assumed heterogeneous system with possibility of having $M$ rates with $N$ SER values, we formulate the average required transmit power as

$$
E\left\{P_{x}\right\}=\sum_{m, n=1}^{M, N} P_{m, n} \int_{0}^{\infty} \frac{\gamma_{m, n}}{\gamma} f(\gamma) \cdot d \gamma,
$$

where $P_{m, n}$ is the probability that the QoS guarantee of the scheduled user is satisfied by $\mathrm{SER}_{n}$ and $R_{m}$. Using the power series expansion and integration by parts for (7), (10) can be solved in a closed-form expression as (see Appendix A):

$$
\begin{aligned}
E\left\{P_{x}\right\}= & \sum_{m, n=1}^{M, N} P_{m, n} \gamma_{m, n} \sigma^{2} K \\
& \times \sum_{k=0}^{K-1}\left(\begin{array}{c}
K-1 \\
k
\end{array}\right)(-1)^{k} \ln \left(\sigma^{2}(k+1)\right) .
\end{aligned}
$$

\section{Power Saving under Resources Outage}

The previous section introduced a power management technique that can perfectly fulfill the QoS requirements for all users with the lowest amount of transmit power. In commercial systems, the transmit power is limited to a maximum value (e.g., 1 Watt) defined by the corresponding standard. Such a limiting factor will definitely affect our proposal, so in this section we deal with the power limitation problem and we obtain its probability through mathematical expressions. We then present the amount of saved power under the maximum power restriction.

4.1. Resource Outage Probability. If the required power $P_{x}$ is lower than the total available power $P_{T}$, obviously the
QoS will be satisfied and we can apply the power saving proposed algorithm described in the previous section. On the other hand, the amount of required transmit power $P_{x}$ that satisfies the required QoS may be larger than the maximum allowed power $P_{T}$ by the system. Therefore, the QoS will not be satisfied and the user suffers from a power outage. By referring to (9), the power outage occurs only if the measured SNR $(\gamma)$ is less than the required modulation threshold $\left(\gamma_{m, n}\right)$ within the demanded modulation region (i.e., corresponding to the demanded rate and SER values). The probability of power outage equals to the integral of the PDF of the serving SNR from zero to the threshold of the required modulation $\gamma_{m, n}$ multiplied by the probability of the modulation threshold. Mathematically, such resource power outage probability $\left(\operatorname{Pr}_{o}\right)$ we give it by

$$
\operatorname{Pr}_{o}=\sum_{m, n=1}^{M, N} P_{m, n} \int_{0}^{\gamma_{m, n}} f(\gamma) \cdot d \gamma=\sum_{m, n=1}^{M, N} P_{m, n} F\left(\gamma_{m, n}\right)
$$

which will be employed in later formulations.

4.2. Saved Power under the Power Outage. Once the probability of the power resource outage has been calculated, we present the amount of saved power, when the proposed technique is applied. These calculations are very useful to the system designer to precisely adjust the power supply at the BS.

The amount of saved power $P_{s}$ is defined as the difference between the total available power $P_{T}$ and the actual transmitted power $P_{x}$, when the measured SNR is larger than the required modulation threshold (i.e., no outage). Therefore, the saved power $P_{s}$ is given by

$$
P_{s}=P_{T}-P_{T} \frac{\gamma_{m, n}}{\gamma} \text { for } \gamma \geq \gamma_{m, n}
$$

and the average saved power over all AM regions for the $N$ SER values and $M$ rates is given by

$$
E\left\{P_{s}\right\}=\sum_{m, n=1}^{M, N} P_{m, n} P_{T} \int_{\gamma_{m, n}}^{\infty}\left(1-\frac{\gamma_{m, n}}{\gamma}\right) f(\gamma) \cdot d \gamma
$$

where the integration starts from $\gamma_{m, n}$ to overcome the probability of outage. Equation (14) can be solved in a closed-form expression and we present it as follows (see Appendix B):

$$
\begin{aligned}
E\left\{P_{s}\right\}=\sum_{m, n=1}^{M, N} P_{m, n} P_{T}[ & 1-F\left(\gamma_{m, n}\right)-K \sigma^{2} \gamma_{m, n} \\
& \times \sum_{k=0}^{K-1}\left(\begin{array}{c}
K-1 \\
k
\end{array}\right)(-1)^{k} E_{1} \\
& \left.\times\left(\sigma^{2} \gamma_{m, n}(k+1)\right)\right],
\end{aligned}
$$

where $E_{1}$ is the exponential integral function defined as $E_{1}(x)=\int_{x}^{\infty}\left(e^{-t} / t\right) \cdot d t[18]$. 


\section{Modified Opportunistic Beamforming}

The QoS satisfaction is the main objective of our power management proposal. However, the power limitation in real systems is the main problem that affects our proposal. Therefore, we propose a method to overcome this problem and minimize the power outage probability. It is based on a modified users scheduling rather than the opportunistic one. Notice that once the user with the best channel characteristics (i.e., with the highest SNR) is selected, (9) indicates that the power outage problem occurs only if the measured SNR is less than the threshold of the required modulation for a predefined QoS satisfaction.

If another user is scheduled with another SNR value lower than the maximum and with lower QoS demands, there exists the probability that the BS could satisfy its demands. Therefore, a new scheduling strategy is now proposed based on such philosophy.

Each user knows its measured SNR value as well as its QoS demands. Therefore, only the users that can satisfy their QoS with their respective SNR values are allowed to feedback. These users can be called the "QoS potential users." Hence, the modified opportunistic beamforming is performed only among the QoS potential users, and not over all the users. Such modified strategy will reduce the feedback load, it avoids the power outage, and it guarantees that the BS will always be able to apply the power saving mechanism.

The probability that a user feeds his SNR back to the BS (i.e., being a QoS potential user) $\left(\operatorname{Pr}_{f}\right)$ is defined as the probability that the required power is lower than the total available power at the BS. We formulate it as

$$
\operatorname{Pr}_{f}=\sum_{m, n=1}^{M, N} P_{m, n} \int_{\gamma_{m, n}}^{\infty} b(\gamma) \cdot d \gamma
$$

which can be simplified through (5) as

$$
\operatorname{Pr}_{f}=1-\sum_{m, n=1}^{M, N} P_{m, n} B\left(\gamma_{m, n}\right) .
$$

The feedback load $F_{L}$ is defined as the number of feedback users $k$ out of the total number of users $K$, which follows the binomial distribution as

$$
F_{L}=\left(\begin{array}{l}
K \\
k
\end{array}\right)\left(\operatorname{Pr}_{f}\right)^{k}\left(1-\operatorname{Pr}_{f}\right)^{K-k},
$$

and the average number of feedback users $\bar{K}$ can be given as

$$
\bar{K}=K \cdot \operatorname{Pr}_{f} .
$$

To show the amount of saved power using this modified scheduling, we must obtain the cdf representing the modified serving SNR based in this proposal. The cdf of the $k$ th maximum user can be easily derived using the order statistics cdf, considering that the cdf of the $k$ th maximum user is the cdf of the $(K-k)$ th order statistics [15]. Hence, the $k$ th maximum user has a modified cdf obtained as

$$
F^{(k)}(\gamma)=\sum_{j=0}^{k-1}\left(\begin{array}{c}
K \\
j
\end{array}\right)(B(\gamma))^{K-j}(1-B(\gamma))^{j} .
$$

The amount of saved power can be derived by referring to (14) and replacing the pdf $f(\gamma)$ by a modified pdf $f^{(k)}$ representing the new serving SNR, which we obtain by

$$
\begin{aligned}
E\left\{P_{s}^{*}\right\}= & \sum_{m, n=1}^{M, N} P_{m, n} P_{T} \\
& \times \sum_{k=1}^{K} \operatorname{Pr}^{(k)} \int_{\gamma_{m, n}}^{\infty}\left(1-\frac{\gamma_{m, n}}{\gamma}\right) f^{(k)}(\gamma) \cdot d \gamma,
\end{aligned}
$$

where $\operatorname{Pr}^{(k)}$ is the probability that the serving SNR is the $k$ th maximum SNR, which we give as

$$
\operatorname{Pr}^{(k)}=\left(1-F^{(k)}\left(\gamma_{m, n}\right)\right) \prod_{i=1}^{k-1} F^{(i)}\left(\gamma_{m, n}\right)
$$

Equation (21) represents the amount of saved power $P_{S}^{*}$ for the modified opportunistic beamforming scheme. By using the equations in Appendix B, it can be represented in a closed form as

$$
\begin{aligned}
E\left\{P_{s}^{*}\right\}=\sum_{m, n=1}^{M, N} P_{m, n} P_{T} \sum_{k=1}^{K} \operatorname{Pr}^{(k)} & {\left[1-F^{(k)}\left(\gamma_{m, n}\right)\right.} \\
+ & \left.A_{i j k} \sigma^{2} \gamma_{m, n} E_{1}\left(\sigma^{2} \gamma_{m, n}(i+j)\right)\right],
\end{aligned}
$$

where the constant $A_{i j k}$ is defined through

$$
A_{i j k}=\sum_{i=0}^{k-1} \sum_{j=0}^{K-i}\left(\begin{array}{c}
K \\
i
\end{array}\right)\left(\begin{array}{c}
K-i \\
j
\end{array}\right)(-1)^{j}(i+j) .
$$

Notice that applying this modified scheduling leads to zero power-outage probability. It is worth noting that there is a small probability of not serving any user. This probability occurs when all users cannot satisfy their QoS demands, and hence, none of the users will feed back its SNR. To get that probability, we substitute $(k=0)$ in (18) as follows:

$$
P_{\text {no }}=\left(1-\operatorname{Pr}_{f}\right)^{K} \text {. }
$$

\section{Simulations}

To assess the performance of the proposed power management technique, a heterogeneous scenario is set up where users with different QoS requirements coexist in the system. The results are obtained by Monte Carlo simulations. In the considered scenario, the BS intends to communicate with a single user at a time. A total of $K=10$ users are available in the system with i.i.d. channel characteristics. A noise variance of $\sigma^{2}=1$ is also assumed together with a total antenna gain at transmitter and receiver of $15 \mathrm{dBi}$. At the $\mathrm{BS}$, after all users report their SNRs, the user with the maximum SNR is scheduled and the power saving mechanism is then applied. Table 2 presents the assumed QoS requirements for each user, and the mapping between these different QoS requirements 
TABLE 2: QoS requirements and SNR thresholds for all users.

\begin{tabular}{lccc}
\hline User index & SER & $\begin{array}{c}\text { Modulation } \\
\text { type }\end{array}$ & $\begin{array}{c}\text { SNR threshold } \gamma_{m, n} \\
(\mathrm{~dB})\end{array}$ \\
\hline User 1 & $10^{-3}$ & BPSK & 6.79 \\
User 2 & $10^{-3}$ & QPSK & 10.34 \\
User 3 & $10^{-4}$ & QPSK & 11.80 \\
User 4 & $10^{-3}$ & 8QAM & 14.21 \\
User 5 & $10^{-3}$ & 8QAM & 14.21 \\
User 6 & $10^{-4}$ & 8QAM & 15.62 \\
User 7 & $10^{-5}$ & 8QAM & 16.69 \\
User 8 & $10^{-3}$ & 16QAM & 17.62 \\
User 9 & $10^{-4}$ & 16QAM & 19.00 \\
User 10 & $10^{-5}$ & 16QAM & 20.06 \\
\hline
\end{tabular}

and the minimum required SNR for QoS guarantee. The calculation of these values is based on the SER equations in [17]. In theoretical results, since we assume i.i.d users, the probability of serving a user with specific QoS requirements is equal among users; that is, $P_{m, n}$ is equal for all users.

The average amount of required transmit power versus the average SNR of the system is shown in Figure 1. Remember that the required transmit power is the amount of power that can satisfy the QoS for any user. Figure 1 shows that the average required power decreases for an increasing SNR value because as the average scheduled SNR increases, the required power to satisfy a QoS demand will decrease from (9). Also from Figure 1, notice that the required transmitted power decreases with the number of users. The multiuser gain has always been presented to enhance the average data rate of the system [5], while here we see in our case that it can be also employed to achieve a great power reduction in the system. Comparing the results obtained by simulation to the mathematical expression that we previously formulated in (11), we notice the exact match between them as the obtained mathematical results were not based on approximation but on the exact system pdf and cdf expressions.

Regarding the power limitation fact in practical systems, our proposed technique generates outage in the power resource. Figure 2 shows the probabilities of power outage versus the average SNR for 10 and 20 users. As the power outage occurs only if the required power is larger than the available power, while the required transmit power decreases for an increasing SNR value, then the power outage probability will consequently decrease as the SNR value enlarges, as confirmed in Figure 2. Here also, the multiuser diversity provides helpful properties to the system, as it enhances the reduction in the power outage probabilities. Notice the exact match between the simulation and the theoretical results (see (12)), which support our previously presented theoretical analysis.

To overcome the power outage problem, a modified opportunistic beamforming scheme was proposed in Section 5. Following this proposed algorithm, Figure 3 plots the percentage of saved power versus the average SNR. Notice that the percentage of saved power reaches up to $95 \%$ for

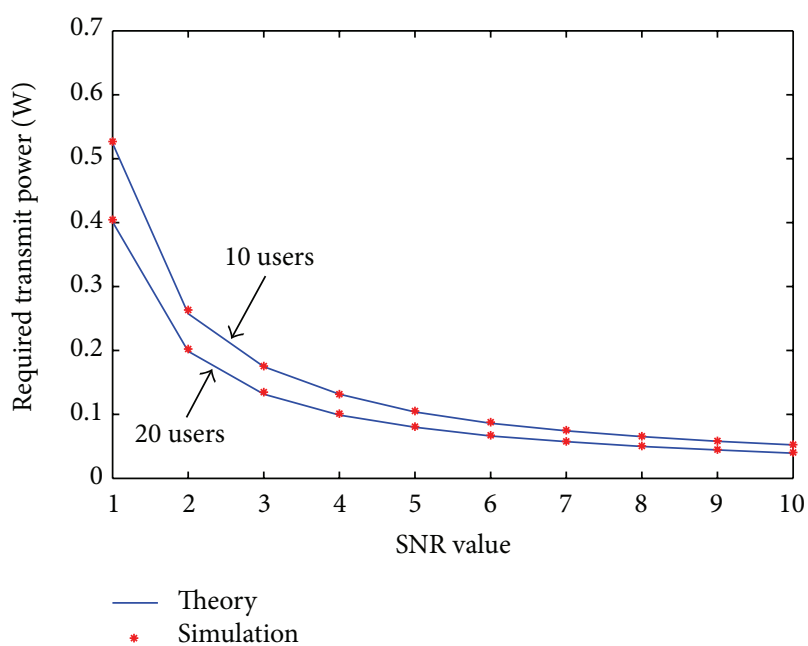

FIGURE 1: The required transmit power versus the average SNR.

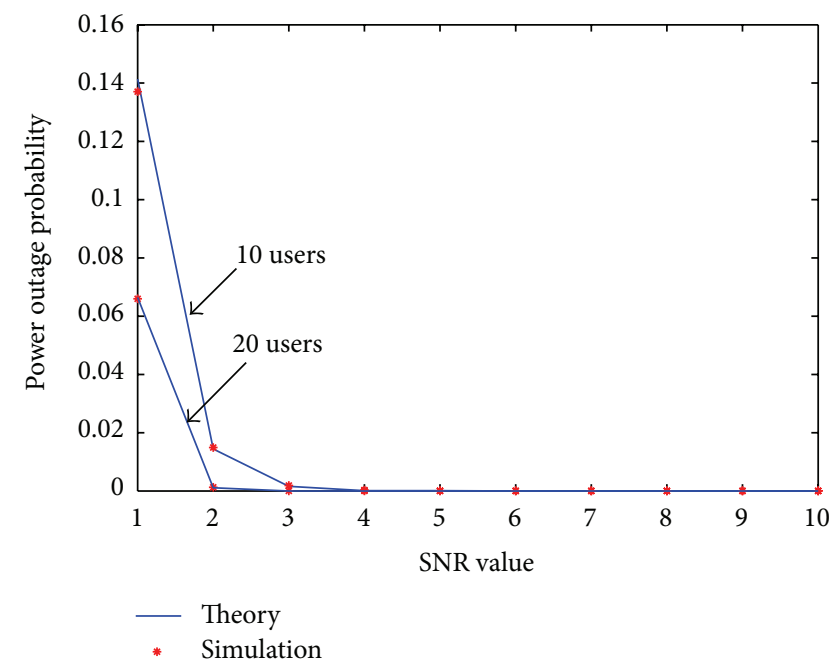

FIGURE 2: The probability of power outage versus the average SNR.

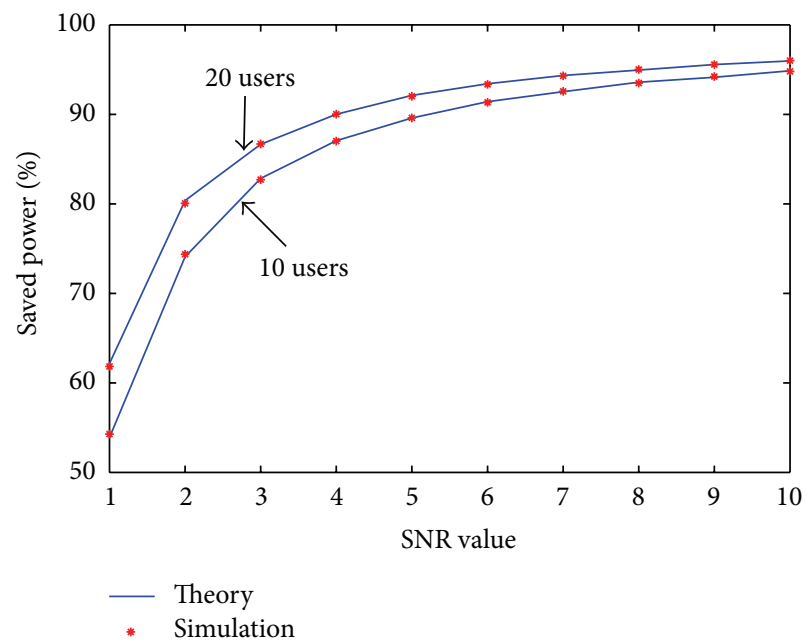

FIGURE 3: The percentage of saved power versus the average SNR by applying the modified opportunistic beamforming. 
high SNR values, showing the large power saving that can be obtained by allowing feedback only to the users who could satisfy their QoS demands. The results are plotted for 10 and 20 users to show the multiuser gain within our proposal. It is worthy to mention that our proposed scheduling always requires an equal or smaller transmitted power compared to the classical opportunistic beamforming. This is because the proposed scheduling serves users with lower QoS requirements. The analytical results are obtained using (23).

\section{Conclusions}

The paper proposes a cross-layer power management strategy that benefits from the Adaptive Modulation intervals, where a quality of service indicator is defined in terms of the minimum data rate with the maximum allowed SER value. The transmitted power is decreased to the minimum required level to match the QoS demands while a multiuser scheduling is used to select the user with the best channel characteristics at each time instant. Closed-form mathematical expressions are obtained for the amounts of transmitted and saved power. The power limitation problem is also considered, where the power resource outage probability is introduced in a closedform expression. A modified opportunistic beamforming is proposed to overcome the power resource outage problem in practical systems. Simulations show an exact match with the theoretical expressions. The system multiuser gain is presented as a potential resource to enhance the power efficiency of the system.

The analysis of the proposed mechanism of power management sheds light on more advanced mechanisms to be applied for Multiple-Input Multiple-Output (MIMO) Beamforming strategies [19] and MIMO Relay [20] scenarios, as the same philosophy of power management could be applied.

\section{Appendices}

\section{A. Derivation of (11)}

Using (7), we can rewrite (10) as

$$
\begin{aligned}
E\left\{P_{x}\right\}=\sum_{m, n=1}^{M, N} P_{m, n}\left[\int_{0}^{\infty} \frac{K \sigma^{2} \gamma_{m, n} e^{-\sigma^{2} \gamma}}{\gamma}\right. & \\
& \left.\times\left(1-e^{-\sigma^{2} \gamma}\right)^{K-1} \cdot d \gamma\right] .
\end{aligned}
$$

Using the binomial power series, we write

$$
\begin{aligned}
E\left\{P_{x}\right\}=\sum_{m, n=1}^{M, N} P_{m, n}\left[\int_{0}^{\infty} \frac{K \sigma^{2} \gamma_{m, n}}{\gamma}\right. & \\
& \left.\times \sum_{k=0}^{K-1}\left(\begin{array}{c}
K-1 \\
k
\end{array}\right)(-1)^{k} e^{-\sigma^{2} \gamma(k+1)} \cdot d \gamma\right],
\end{aligned}
$$

which we rearrange as

$$
\begin{aligned}
E\left\{P_{x}\right\}=\sum_{m, n=1}^{M, N} P_{m, n} & {\left[K \sigma^{2} \gamma_{m, n}\right.} \\
& \left.\times \sum_{k=0}^{K-1}\left(\begin{array}{c}
K-1 \\
k
\end{array}\right)(-1)^{k} \int_{0}^{\infty} \frac{e^{-\sigma^{2} \gamma(k+1)}}{\gamma} \cdot d \gamma\right] .
\end{aligned}
$$

The integral can be divided into two integrals as follows:

$$
\begin{array}{r}
E\left\{P_{x}\right\}=\sum_{m, n=1}^{M, N} P_{m, n}\left[K \sigma^{2} \gamma_{m, n} \times \sum_{k=0}^{K-1}\left(\begin{array}{c}
K-1 \\
k
\end{array}\right)(-1)^{k}\right. \\
\times\left(\int_{0}^{1} \frac{e^{-\sigma^{2} \gamma(k+1)}}{\gamma} \cdot d \gamma\right. \\
\left.\left.+\int_{1}^{\infty} \frac{e^{-\sigma^{2} \gamma(k+1)}}{\gamma} \cdot d \gamma\right)\right],
\end{array}
$$

where we replace the second integral by the exponential integral, defined as $E_{1}(x)=\int_{1}^{\infty}\left(e^{-x t} / t\right) \cdot d t$ [18], so that our last expression becomes

$$
\begin{array}{r}
E\left\{P_{x}\right\}=\sum_{m, n=1}^{M, N} P_{m, n}\left[K \sigma^{2} \gamma_{m, n} \times \sum_{k=0}^{K-1}\left(\begin{array}{c}
K-1 \\
k
\end{array}\right)(-1)^{k}\right. \\
\times\left(\int_{0}^{1} \frac{e^{-\sigma^{2} \gamma(k+1)}}{\gamma} \cdot d \gamma\right. \\
\left.\left.+E_{1}\left(\sigma^{2}(k+1)\right)\right)\right] .
\end{array}
$$

It remains to solve the first integral which is an improper integral, using the power series representation of the exponential function, we rewrite it as follows:

$$
\begin{array}{r}
E\left\{P_{x}\right\}=\sum_{m, n=1}^{M, N} P_{m, n}\left[K \sigma^{2} \gamma_{m, n} \times \sum_{k=0}^{K-1}\left(\begin{array}{c}
K-1 \\
k
\end{array}\right)(-1)^{k}\right. \\
\times\left(\int_{0}^{1} \sum_{i=0}^{\infty} \frac{\left(-\sigma^{2}(k+1) \gamma\right)^{i}}{i ! \gamma} \cdot d \gamma\right. \\
\left.\left.+E_{1}\left(\sigma^{2}(k+1)\right)\right)\right],
\end{array}
$$


that is simplified as

$$
\begin{aligned}
E\left\{P_{x}\right\}=\sum_{m, n=1}^{M, N} P_{m, n} & {\left[K \sigma^{2} \gamma_{m, n}\right.} \\
& \times\left(\int_{0}^{1} \sum_{i=0}^{\infty} \frac{\left(-\sigma^{2}(k+1)\right)^{i}(\gamma)^{i-1}}{i !} \cdot d \gamma\right. \\
& \left.\left.+E_{1}\left(\sigma^{2}(k+1)\right)\right)\right] .
\end{aligned}
$$

Performing the integration, we obtain

$$
\begin{aligned}
& E\left\{P_{x}\right\}=\sum_{m, n=1}^{M, N} P_{m, n}\left[K \sigma^{2} \gamma_{m, n} \times \sum_{k=0}^{K-1}\left(\begin{array}{c}
K-1 \\
k
\end{array}\right)(-1)^{k}\right. \\
& \times \sum_{k=0}^{K-1}\left(\begin{array}{c}
K-1 \\
k
\end{array}\right)(-1)^{k} \\
& \times\left(\left.\sum_{i=0}^{\infty} \frac{\left(-\sigma^{2}(k+1)\right)^{i} \gamma^{i}}{i \cdot i !}\right|_{0} ^{1}\right. \\
& \left.\left.+E_{1}\left(\sigma^{2}(k+1)\right)\right)\right]
\end{aligned}
$$

that by substituting the integral limits, we state it as

$$
\begin{aligned}
E\left\{P_{x}\right\}=\sum_{m, n=1}^{M, N} P_{m, n} & {\left[K \sigma^{2} \gamma_{m, n} \times \sum_{k=0}^{K-1}\left(\begin{array}{c}
K-1 \\
k
\end{array}\right)(-1)^{k}\right.} \\
& \times\left(\sum_{i=0}^{\infty} \frac{\left(-\sigma^{2}(k+1)\right)^{i}}{i \cdot i !}\right. \\
& \left.\left.+E_{1}\left(\sigma^{2}(k+1)\right)\right)\right] .
\end{aligned}
$$

The first term $(i=0)$ in the third summation is constant, through the identity $\sum_{h=0}^{H}\left(\begin{array}{c}H \\ h\end{array}\right)(-1)^{h} a=0$ when $a=$ constant [18], we reformulate the last equation as

$$
\begin{aligned}
E\left\{P_{x}\right\}=\sum_{m, n=1}^{M, N} P_{m, n} & {\left[K \sigma^{2} \gamma_{m, n} \times \sum_{k=0}^{K-1}\left(\begin{array}{c}
K-1 \\
k
\end{array}\right)(-1)^{k}\right.} \\
& \times\left(\sum_{i=1}^{\infty} \frac{\left(-\sigma^{2}(k+1)\right)^{i}}{i \cdot i !}\right. \\
& \left.\left.+E_{1}\left(\sigma^{2}(k+1)\right)\right)\right]
\end{aligned}
$$

where we evaluate the last summation as follows:

$$
\begin{array}{rl}
E\left\{P_{x}\right\}=\sum_{m, n=1}^{M, N} P_{m, n} & K \sigma^{2} \gamma_{m, n} \\
& \times \sum_{k=0}^{K-1}\left(\begin{array}{c}
K-1 \\
k
\end{array}\right)(-1)^{k} \\
& \times\left(-\delta-\ln \left(\sigma^{2}(k+1)\right)-E_{1}\left(\sigma^{2}(k+1)\right)\right. \\
& \left.\left.+E_{1}\left(\sigma^{2}(k+1)\right)\right)\right],
\end{array}
$$

where $\delta$ is Euler constant. Using the previous identity where $\delta$ is a constant, we obtain the amount of required transmit power as

$$
\begin{aligned}
E\left\{P_{x}\right\}= & \sum_{m, n=1}^{M, N} P_{m, n} K \sigma^{2} \gamma_{m, n} \\
& \times \sum_{k=0}^{K-1}\left(\begin{array}{c}
K-1 \\
k
\end{array}\right)(-1)^{k}\left(\ln \left(\sigma^{2}(k+1)\right)\right)
\end{aligned}
$$

as given in (11).

\section{B. Derivation of (15)}

We expand the integral of (14) into two integrals as follows:

$$
\begin{aligned}
E\left\{P_{s}\right\}= & \sum_{m, n=1}^{M, N} P_{m, n} P_{T} \\
& \times\left[\int_{\gamma_{m, n}}^{\infty} f(\gamma) \cdot d \gamma-\int_{\gamma_{m, n}}^{\infty} \frac{\gamma_{m, n}}{\gamma} f(\gamma) \cdot d \gamma\right],
\end{aligned}
$$

where the first integral equals to $1-F\left(\gamma_{m, n}\right)$. Using (7), we rewrite it as

$$
\begin{aligned}
E\left\{P_{s}\right\}=\sum_{m, n=1}^{M, N} P_{m, n} P_{T} & {\left[1-F\left(\gamma_{m, n}\right)\right.} \\
& \left.-\int_{\gamma_{m, n}}^{\infty} \frac{K \sigma^{2} \gamma_{m, n}}{\gamma} e^{-\sigma^{2} \gamma}\left(1-e^{-\sigma^{2} \gamma}\right)^{K-1} \cdot d \gamma\right] .
\end{aligned}
$$

Using power series expansion, we simplify (B.2) as

$$
\begin{aligned}
E\left\{P_{s}\right\}=\sum_{m, n=1}^{M, N} P_{m, n} P_{T}[ & 1-F\left(\gamma_{m, n}\right)-\int_{\gamma_{m, n}}^{\infty} \frac{K \sigma^{2} \gamma_{m, n}}{\gamma} \\
& \left.\times \sum_{k=0}^{K-1}\left(\begin{array}{c}
K-1 \\
k
\end{array}\right)(-1)^{k} e^{-\sigma^{2}(k+1) \gamma} \cdot d \gamma\right],
\end{aligned}
$$


which we rearrange as

$$
\begin{aligned}
E\left\{P_{s}\right\}=\sum_{m, n=1}^{M, N} P_{m, n} P_{T} & {\left[1-F\left(\gamma_{m, n}\right)-K \sigma^{2} \gamma_{m, n}\right.} \\
& \left.\times \sum_{k=0}^{K-1}\left(\begin{array}{c}
K-1 \\
k
\end{array}\right)(-1)^{k} \int_{\gamma_{m, n}}^{\infty} \frac{e^{-\sigma^{2}(k+1) \gamma}}{\gamma} \cdot d \gamma\right] .
\end{aligned}
$$

Using the exponential integral [18], we provide the amount of saved power as the closed-form expression

$$
\begin{aligned}
E\left\{P_{s}\right\}=\sum_{m, n=1}^{M, N} P_{m, n} P_{T} & {\left[1-F\left(\gamma_{m, n}\right)-K \sigma^{2} \gamma_{m, n}\right.} \\
& \left.\times \sum_{k=0}^{K-1}\left(\begin{array}{c}
K-1 \\
k
\end{array}\right)(-1)^{k} E_{1}\left(\sigma^{2} \gamma_{m, n}(k+1)\right)\right]
\end{aligned}
$$

as given in (15).

\section{Acknowledgments}

This work has been funded by the Research Projects GREENET (PITN-GA-2010-264759), CO2GREEN (TEC2010-20823), and GREEN-T (CP8-006).

\section{References}

[1] W. Vereecken, W. Van Heddeghem, M. Deruyck et al., "Power consumption in telecommunication networks: Overview and reduction strategies," IEEE Communications Magazine, vol. 49, no. 6, pp. 62-69, 2011.

[2] J. Choi and J. Ha, "On the energy efficiency of AMC and HARQIR with QoS constraints," IEEE Transactions on Vehicular Technology, vol. 99, no. 1, 2013.

[3] N. Zorba, A. I. Pérez-Neira, A. Foglar, and C. Verikoukis, "Cross layer qoS guarantees in multiuser wlan systems," Wireless Personal Communications, vol. 51, no. 3, pp. 549-563, 2009.

[4] P. Svedman, S. K. Wilson, L. J. Cimini Jr., and B. Ottersten, "Opportunistic beamforming and scheduling for OFDMA systems," IEEE Transactions on Communications, vol. 55, no. 5, pp. 941-952, 2007.

[5] T. Samarasinghe, H. Inaltekin, and J. Evans, "Optimal selective feedback policies for opportunistic beamforming," IEEE Transactions on Information Theory, vol. 59, no. 5, 2013.

[6] C.-H. Choi, H.-J. Lim, T.-K. Kim, G.-H. Im, and V. B. Lawrence, "Spectral efficient multiuser technique with channel-dependent resource allocation schemes," IEEE Transactions on Wireless Communications, vol. 11, no. 3, pp. 990-999, 2012.

[7] S. Bashar and Z. Ding, "Admission control and resource allocation in a heterogeneous OFDMA wireless network," IEEE Transactions on Wireless Communications, vol. 8, no. 8, pp. 4200-4210, 2009.

[8] G. I. Tsiropoulos, D. G. Stratogiannis, P. G. Cottis, T. D. Lagkas, and P. Chatzimisios, "Adaptive resource allocation and dynamic call admission control in wireless networks," in Proceedings of the IEEE Global Communications Conference (GLOBECOM '10), pp. 1217-1221, Miami, Fla, USA, December 2010.
[9] C. W. Leong, W. Zhuang, Y. Cheng, and L. Wang, "Optimal resource allocation and adaptive call admission control for voice/data integrated cellular networks," IEEE Transactions on Vehicular Technology, vol. 55, no. 2, pp. 654-669, 2006.

[10] N. Zorba and A. I. Pérez-Neira, "Robust power allocation schemes for multibeam opportunistic transmission strategies under quality of service constraints," IEEE Journal on Selected Areas in Communications, vol. 26, no. 6, pp. 1025-1034, 2008.

[11] C.-F. Tsai, C.-J. Chang, F.-C. Ren, and C.-M. Yen, "Adaptive radio resource allocation for downlink OFDMA/SDMA systems with multimedia traffic," IEEE Transactions on Wireless Communications, vol. 7, no. 5, pp. 1734-1743, 2008.

[12] Y. Long, H. Li, M. Pan, Y. Fang, and T. F. Wong, "A fair QoSaware resource allocation scheme for multi-radio multi-channel networks," IEEE Transactions on Vehicular Technology, no. 99, 2013.

[13] K. Son, B. C. Jung, S. Chong, and D. K. Sung, "Power allocation for OFDM-based cognitive radio systems under outage constraints," in Proceedings of the IEEE International Conference on Communications (ICC '10), Cape Town, South Africa, May 2010.

[14] L. Yang and M.-S. Alouini, "Performance analysis of multiuser selection diversity," in Proceedings of the IEEE International Conference on Communications, pp. 3066-3070, Paris, France, June 2004.

[15] B. C. Arnold, N. Balakrishnan, and H. N. Nagaraja, A First Course in Order Statistics, John Wiley \& Sons, 1992.

[16] R. Fantacci, D. Marabissi, D. Tarchi, and I. Habib, "Adaptive modulation and coding techniques for OFDMA systems," IEEE Transactions on Wireless Communications, vol. 8, no. 9, pp. 4876-4883, 2009.

[17] J. Proakis, Digital Communications, McGraw-Hill, New York, NY, USA, 4th edition, 2000.

[18] I. S. Gradshteyn and I. M. Ryzhik, Table of Integrals, Series, and Products, Academic Press, New York, NY, USA, 4th edition, 1994.

[19] A. Kalis, A. G. Kanatas, and G. P. Efthymoglou, "A co-operative beamforming solution for eliminating multi-hop communications in wireless sensor networks," IEEE Journal on Selected Areas in Communications, vol. 28, no. 7, pp. 1055-1062, 2010.

[20] E. T. Michailidis, P. Theofylakos, and A. G. Kanatas, "Threedimensional modeling and simulation of MIMO mobile-tomobile via stratospheric relay fading channels," IEEE Transactions on Vehicular Technology, vol. 62, no. 5, pp. 2014-2030, 2012. 

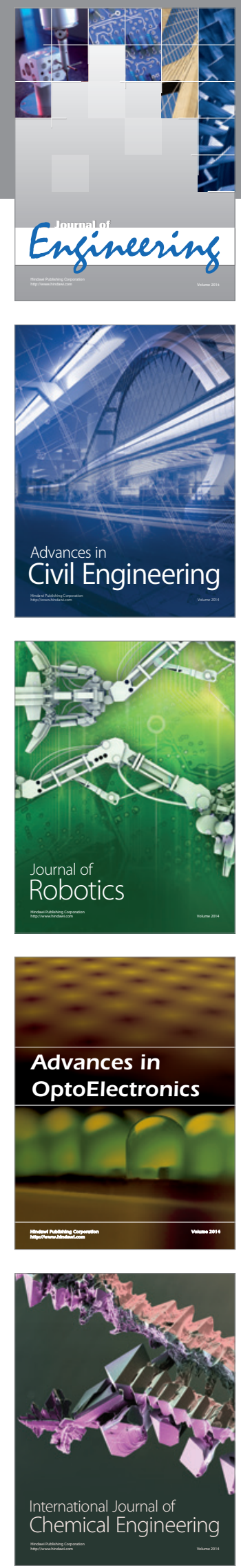

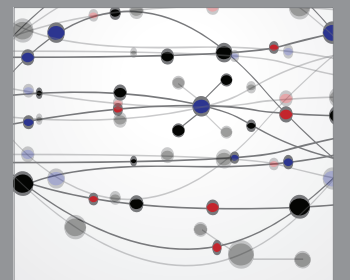

The Scientific World Journal
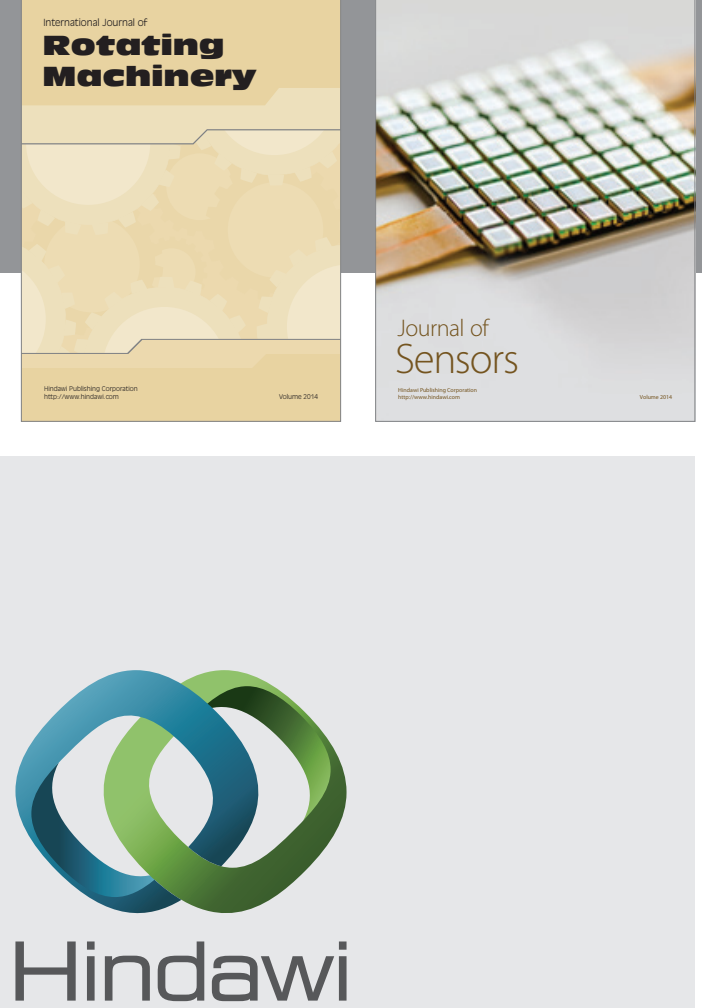

Submit your manuscripts at http://www.hindawi.com
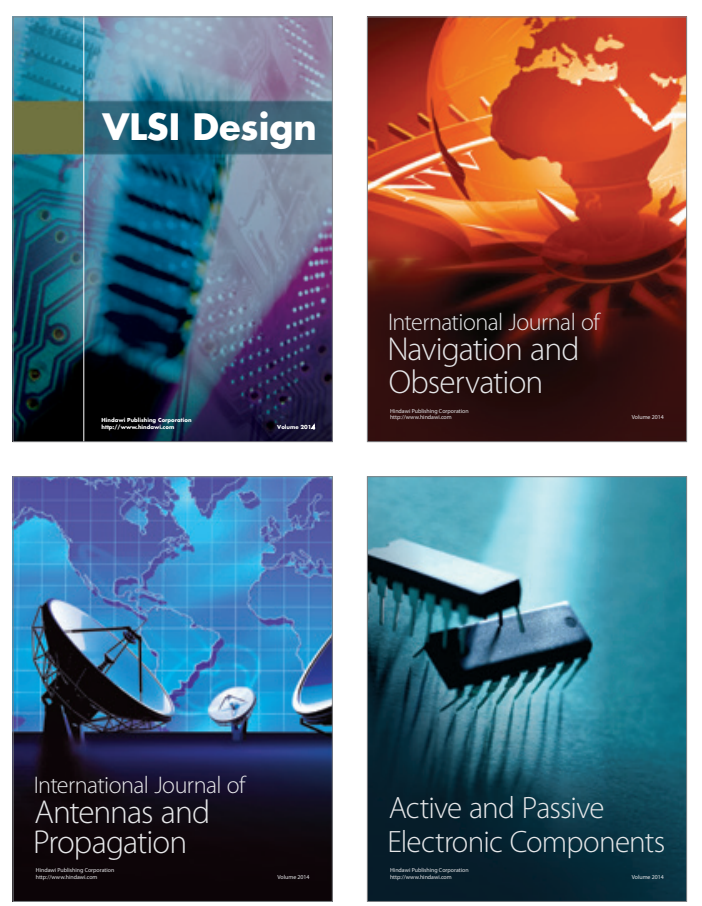
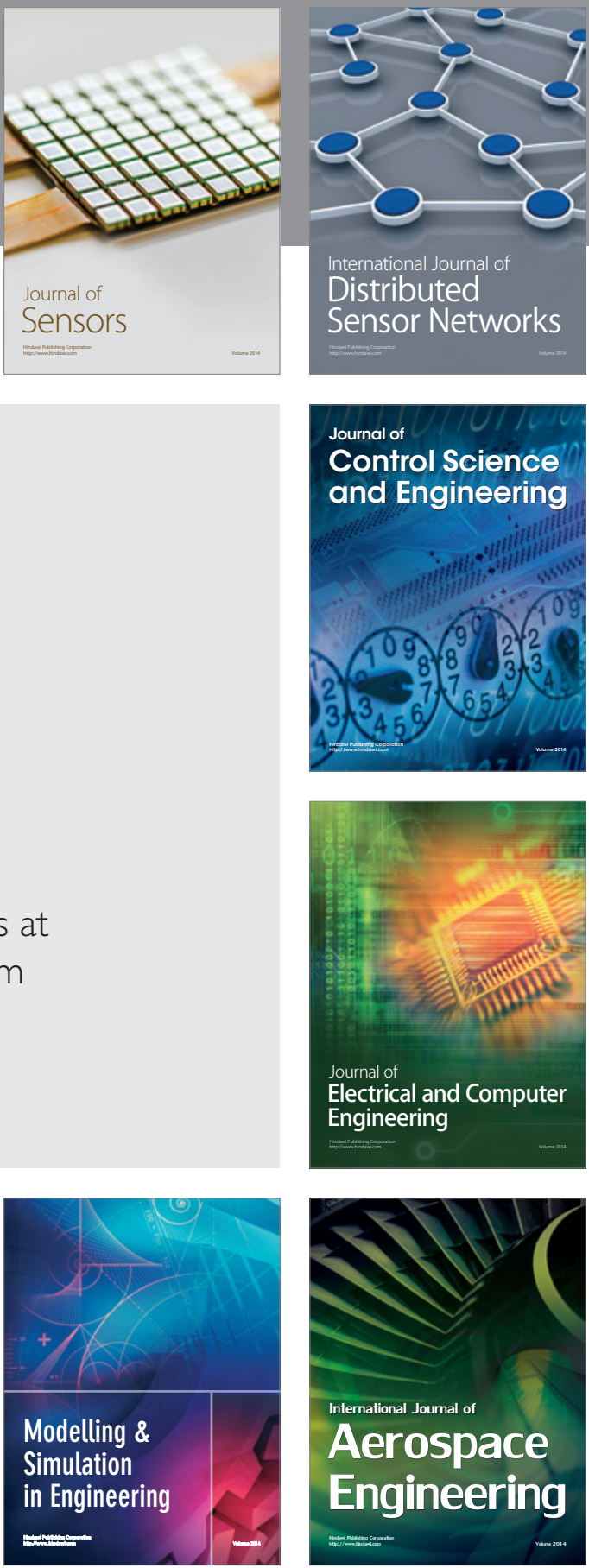

Journal of

Control Science

and Engineering
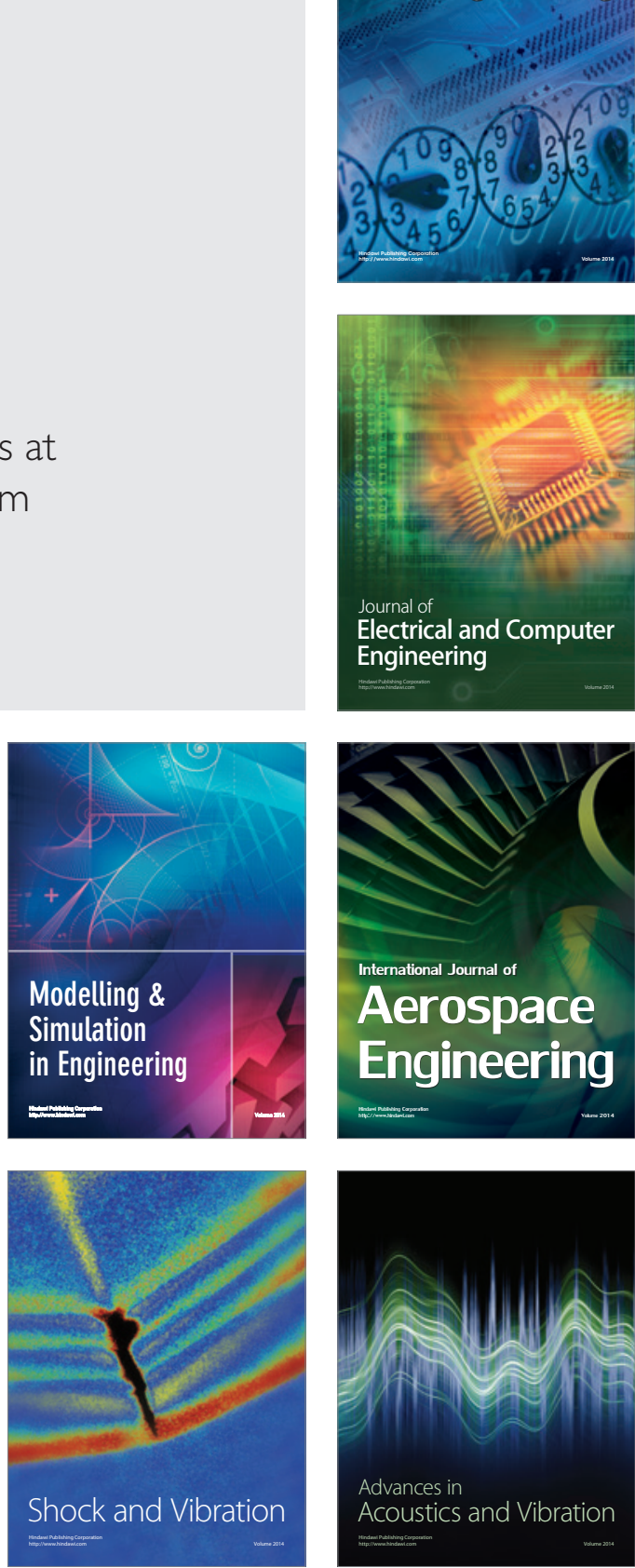